\title{
Are Nouns Learned Before Verbs? Infants Provide Insight Into a Long-Standing Debate
}

\author{
Sandra Waxman, ${ }^{1}$ Xiaolan Fu, ${ }^{2}$ Sudha Arunachalam, ${ }^{3}$ Erin Leddon, ${ }^{1}$ Kathleen Geraghty, ${ }^{1}$ \\ and Hyun-joo Song ${ }^{4}$
}

${ }^{1}$ Northwestern University, ${ }^{2}$ Chinese Academy of Sciences, ${ }^{3}$ Boston University, and ${ }^{4}$ Yonsei University

ABSTRACT_For decades, a spirited debate has existed over whether infants' remarkable capacity to learn words is shaped primarily by universal features of human language or by specific features of the particular native language they are acquiring. A strong focus for this debate has been a well-documented difference in early word learning: Infants' success in learning verbs lags behind their success in learning nouns. In this review, we articulate both sides of the debate and summarize new cross-linguistic evidence from infants that underscores the role of universal features and begins to clarify the impact of distinctly different languages on early language and conceptual development.

KEYWORDS_language development; cognitive development; cross-linguistic studies; infancy

This review engages a long-standing theoretical and empirical debate concerning whether and how the acquisition of nouns and verbs - two fundamental grammatical forms that are expressed universally across the worlds' languages — is shaped

Sandra Waxman, Department of Psychology, Northwestern University; Xiaolan Fu, Institute of Psychology, Chinese Academy of Sciences (Beijing); Sudha Arunachalam, Department of Applied Linguistics, Boston University; Erin Leddon, Department of Linguistics, Northwestern University; Kathleen Geraghty, Department of Psychology, Northwestern University; Hyun-joo Song, Department of Psychology, Yonsei University (Seoul).

This work was supported by NIH HD30410 and NSF BCS 1023300 to SRW. We are grateful to our colleagues and collaborators for the insights they have provided in discussions of this review.

Correspondence concerning this article should be addressed to Sandra R. Waxman, Department of Psychology, Swift Hall, Northwestern University, 2029 Sheridan Rd., Evanston, IL 60208-2710; e-mail: s-waxman@northwestern.edu.

(C) 2013 The Authors

Child Development Perspectives @ 2013 The Society for Research in Child Development DOI: $10.1111 /$ cdep.12032 by features of the particular language being acquired. Here, we articulate the issues at stake in this debate, offer fresh theoretical insight, and summarize the most recent cross-linguistic evidence from infants. We close by highlighting a promising new research agenda, one aimed at bringing us closer to discovering the impact of distinctly different languages on early language and cognitive development.

The literature on early word learning reveals that although infants are exquisite word learners, their prowess in learning verbs lags far behind their success in learning nouns. This disparity has engendered a long-standing debate centered around the theoretical tug-of-war between the twin engines of human development: our universal endowments and the shaping role of experience. Although evidence from infants acquiring distinctly different languages holds the key to resolving this debate, only recently has such evidence become available.

Cross-linguistic evidence comparing how nouns and verbs are learned has not been entirely absent. Several comprehensive cross-linguistic analyses have identified the proportions of nouns and verbs in children's vocabularies and used these as an index of the relative facility of acquiring these two grammatical forms. But these analyses have counted the nouns and verbs children know; they cannot reveal the facility with which those words were acquired. Moreover, cross-linguistic experiments were designed to compare how children learned novel nouns and verbs in laboratory settings, but until recently have included preschool-age children, who, at 3-5 years, have already acquired scores of nouns and verbs. As a result, long-standing debates about early language and conceptual development remain unresolved.

\section{TWO DIFFERENT THEORETICAL PERSPECTIVES}

The Early Noun Advantage Is Universal

For decades, researchers have asserted that the early advantage for learning nouns over verbs is a universal feature of human language. Indeed, two potential sources of the early noun 
advantage have been identified. Some have attributed it to fundamental differences in the concepts underlying the meaning of nouns and verbs, noting that concepts of objects (universally labeled by nouns) are perceptually and conceptually more stable, and therefore more readily acquired, than concepts of actions or events, which involve relations among objects (labeled by verbs; Gentner, 1982). Consider, for example, a cat scratching a dog and then leaping onto a ledge. Both participant objects (the cat and the dog) are visible throughout the scene, before, during, and after the scratching occurs. In contrast to this stability of the objects, the relation between them (scratching) is more fleeting, observable only in the moment that the scratching takes place. In addition to their stability, the concepts underlying most nouns are more concrete, or imageable, than those underlying most verbs (Gentner, 2006; Gentner \& Boroditsky, 2001; Ma, Golinkoff, Hirsh-Pasek, McDonough, \& Tardif, 2009; McDonough, Song, Hirsh-Pasek, Golinkoff, \& Lannon, 2011).

Others have adopted a different but complementary perspective, attributing the universal early noun advantage to the fundamentally different linguistic requirements underlying how nouns and verbs are learned. Because the meaning of a given verb depends on the arguments (nouns) that it takes, infants may need to establish a repertoire of nouns before they can readily learn verbs. For example, to discover the meaning of the verb scratch, identifying the relevant participant objects (cat; dog) is not enough; the learner must also identify a specific relation between them (agent; patient). (For recent reviews, see Gleitman, Cassidy, Nappa, Papafragou, \& Trueswell, 2005; Golinkoff \& Hirsh-Pasek, 2008; Waxman \& Lidz, 2006).

\section{The Early Noun Advantage Is Language Specific}

Other researchers have proposed a very different view, arguing that the early noun advantage is not a universal feature of human language, but a consequence of the particular language being acquired. Proponents of this language-specific view distinguish between two broad classes of languages: noun-friendly languages (including English and French) in which nouns are said to enjoy a privileged position in the input, and verb-friendly languages (including Mandarin, Korean, Japanese, Inuktitut, Hindi, and Tzeltal) in which verbs are said to enjoy a more privileged position. The claim is that infants' acquisition of nouns and verbs reflects features of the particular language being learned: For infants acquiring noun-friendly languages, nouns outpace verbs in early acquisition, but for infants acquiring verb-friendly languages, the noun advantage is attenuated or absent entirely (Tardif, 1996; Tardif, Gelman, \& Xu, 1999; Tardif, Shatz, \& Naigles, 1997). (For recent reviews, see Bornstein et al., 2004; Imai et al., 2008).

\section{GAPS IN THE LITERATURE}

Two gaps — one theoretical and the other empirical - have until recently limited our ability to adjudicate between these distinct positions. The theoretical gap concerns the status of the assumption that verbs should be more readily acquired in verb-friendly than noun-friendly languages. On one hand, some linguistic features of these languages might offer such an advantage. First, in verb-friendly languages, nouns are typically dropped from the surface of an utterance whenever sufficient support from the context allows the speaker to retrieve their meaning. As a result, nouns are relatively less frequent and verbs more frequent in the input to infants and young children, and verbs can occur alone or occupy the privileged phrase-final position (Choi \& Gopnik, 1995; Kim, McGregor, \& Thompson, 2000; Lee \& Naigles, 2005; Tardif et al., 1997; but see Sandhofer, Smith, \& Luo, 2000). Second, cultural factors may play a role. Some have suggested that in Western cultures, people attend primarily to salient objects (typically marked by nouns), while in Eastern cultures, they attend primarily to relations (typically marked by verbs; Nisbett, Peng, Choi, \& Norenzayan, 2001). This cultural difference may be reflected in interactions involving infants, leading caregivers to engage infants in social routines that emphasize actions and relations rather than those that emphasize objects (Tardif et al., 2008). If speakers of verb-friendly languages focus their infants' attention on verb-relevant information, and if verbs occupy a more prominent position in the input, then verb-friendly languages might confer a relative advantage in verb-learning over noun-friendly languages like English (Gentner, 1982; Tardif et al., 1997; Tardif et al., 2008).

But other linguistic features of verb-friendly languages could actually make learning verbs more difficult. In particular, it may be harder for infants acquiring verb-friendly languages to tease apart nouns from verbs. For example, the English and French languages have rich surface cues that distinguish these major grammatical forms: Nouns and verbs tend to appear in very different linguistic environments (e.g., nouns, but not verbs, tend to follow determiners, e.g., $a$, the), and each grammatical form has distinct morphological properties (e.g., verbs, but not nouns, are marked morphologically for tense, e.g., -ing, -ed endings; Maratsos \& Chalkley, 1980). Moreover, infants are sensitive to these environments and use them to distinguish nouns from verbs (Chemla, Mintz, Bernal, \& Christophe, 2009; Mintz, 2003). But in many verb-friendly languages, such cues are more sparse (Imai et al., 2008). For example, Mandarin has few determiners and scant morphology or other surface cues to distinguish among grammatical forms (Imai, Haryu, \& Okada, 2005; Imai et al., 2008). As a result, teasing the nouns apart from the verbs may be harder for infants acquiring Mandarin than English.

Another linguistic feature of verb-friendly languages may also pose a challenge. We know that young word learners rely on information from the surrounding nouns (e.g., semantic features of the nouns, e.g., animacy, the number of nouns, and their relative positions within the sentence) to discover the meaning of a novel verb (Arunachalam \& Waxman, 2011; Fisher, Hall, Rakowitz, \& Gleitman, 1994; Gleitman, 1990; Gleitman et al., 2005; 
Yuan \& Fisher, 2009). Therefore, because nouns are frequently dropped in verb-friendly languages, infants acquiring these languages may have more difficulty identifying the meaning of a novel verb than in noun-friendly languages.

To adjudicate between the universal and language-specific alternatives, cross-linguistic evidence comparing the acquisition of novel nouns and verbs in infant word learners is essential. Here, we come to the empirical gap: Until recently, no requisite evidence was available. Although analyses of children's vocabulary knowledge (as measured by corpus analyses or by parental report) have been used to evaluate the universality of the noun advantage, they are limited in two ways. First, the results are decidedly mixed, with some demonstrating a robust crosslinguistic noun advantage (Au, Dapretto, \& Song, 1994; Bornstein et al., 2004; Gentner, 1982; Kauschke, Lee, \& Pae, 2007; Kim et al., 2000; Tardif et al., 1999) and others reporting that the noun advantage was either attenuated or absent entirely in verbfriendly languages (Choi, 2000; Choi \& Gopnik, 1995; Gopnik, Choi, \& Baumberger, 1996; Stoll et al., 2012; Tardif, 1996; Tardif et al., 1997; Tardif et al., 1999; Tardif et al., 2008). Second, these analyses cannot address the developmental question at hand. What they offer is essentially a count of the nouns and verbs that a child already produces, not the meaning that they assign to them or the facility with which they were acquired.

To overcome these limitations, researchers designed experiments to compare the acquisition of novel nouns and verbs directly, but the experiments involving infants were conducted almost exclusively in noun-friendly languages, English in particular. These experiments revealed an early noun advantage. Infants acquiring English first identified the nouns in the ambient language and mapped them specifically to objects and object categories; only later, after this noun-category link was firmly in place, did the infants begin to link verbs specifically to event categories (see Waxman \& Lidz, 2006, for a review). Until recently, experiments comparing the acquisition of novel nouns and verbs in noun-friendly and verb-friendly languages involved not infants but 3- to 5-year-olds (Imai et al., 2005; Imai et al., 2008). Although children learning both noun-friendly and verbfriendly languages successfully mapped novel nouns to objects, their difficulties mapping novel verbs to actions were striking (Imai et al., 2005; Imai et al., 2008).

For example, one study compared noun and verb learning in 3- and 5-year-old monolingual children acquiring English, Japanese, or Mandarin (Imai et al., 2005; Imai et al., 2008). All children viewed the same dynamic scenes (e.g., a woman waving a novel object in a novel way) while listening to a novel word that was embedded in linguistic contexts marking them either as nouns (e.g., "This is a blick") or verbs (e.g., "The girl is blicking something"). At test, children viewed two new scenes: In the Same Object scene, the woman used the same object but produced a different action; in the Same Action scene, she produced the same action with a different object. Children acquiring all languages successfully mapped novel nouns to the
Same Object scenes, but found novel verbs much more difficult. Three-year-olds uniformly failed to map novel verbs to the Same Action scenes. Although 5-year-olds succeeded under certain circumstances, their performance was considerably less robust than with nouns. English-acquiring 5-year-olds successfully mapped verbs to actions if the surrounding nouns were mentioned explicitly (e.g., "She is blicking something!") but not if they were dropped (e.g., "Blicking"). In contrast, Japanese 5-year-olds more successfully mapped verbs to actions when the surrounding nouns were dropped than when they were explicitly mentioned. Five-year-olds learning Mandarin failed to map novel verbs to actions in either linguistic context.

This cross-linguistic experimental evidence made three important contributions. First, it revealed that verb-friendly languages may not offer an advantage in acquiring verbs: In all languages, children successfully mapped novel nouns to objects, but had considerable difficulty mapping novel verbs to actions (Imai et al., 2005; Imai et al., 2008). Second, it clarified that not all verb-friendly languages are alike. Finally, it revealed that in word-learning tasks in which an object interpretation is pitted directly against an action interpretation, all children had difficulty mapping verbs to the actions (rather than to the participant objects); this difficulty apparently persists well into the preschool years (Imai et al., 2005; Imai et al., 2008; Kersten \& Smith, 2002; Waxman, Lidz, Braun, \& Lavin, 2009). This is surprising because by age 3 , children have naturally mastered the meaning of scores of verbs and use them fluently.

More recent research reveals that 24-month-old infants acquiring English can map successfully both novel nouns to object categories and novel verbs to event categories (Waxman et al., 2009). Infants viewed several instances of the same dynamic scene (e.g., a man waving a balloon), each time in conjunction with a novel word, presented as either a noun (e.g., "The man is waving the blick") or verb (e.g., "The man is blicking the balloon"). Infants' performance at test revealed that they were influenced by the grammatical form of the novel word. Infants hearing novel nouns focused their attention on test scenes that depicted the same participant objects; those hearing novel verbs focused on scenes depicting the same action. This documented successful noun and verb learning in infants engaged the very process of acquiring their native language.

But evidence from English-acquiring infants alone cannot reveal whether and how features of their ambient language shape the early acquisition of novel nouns and verbs. A handful of recent investigations have begun to provide much-needed evidence from infants acquiring languages other than English.

\section{NEW CROSS-LINGUISTIC EVIDENCE FROM INFANTS}

Even before infants begin to produce verbs in earnest, they have begun to form representations of the kinds of concepts underlying both noun (object-based) and verb (action-based) meaning. For example, in one experiment, infants were shown dynamic 
scenes (e.g., a novel cartoon creature jumping back and forth over a fence) while listening repeatedly to a novel word, presented alone (e.g., "Blick!"; Katerelos, Poulin-Dubois, \& Oshima-Takane, 2011). At test, infants viewed this now-familiar scene and a new scene in which either the participant object changed (e.g., a new creature jumping over a fence) or the action changed (e.g., the same creature now racing across a platform). By 20 months, infants acquiring any of the three languages looked significantly longer at the new scenes in which an element (either the object or the action) changed. Their sensitivity to changes in either the object or action was comparable, suggesting that they appreciated both the object- and actionbased commonalities that underlie noun and verb meaning, respectively. (But see Chan et al., 2011, for evidence from younger infants learning Mandarin.)

To determine whether infants' attention to objects and actions was guided by language, another study focused specifically on the influence of introducing novel verbs (Oshima-Takane, Ariyama, Kobayashi, Katerelos, \& Poulin-Dubois, 2011). Japanese 20month-olds were tested in the same paradigm described above, but this time, the novel word was embedded in a phrase that marked it as a verb (e.g., "It's blicking!"). The Japanese infants now focused specifically on the action, and not the participant object, suggesting that they expected that a verb can be extended to a new scene depicting the same action (e.g., jumping), even if it involves a different object (e.g., a new creature). Thus, for infants learning Japanese, novel verbs appear to direct attention specifically to actions (or to dynamic relations among objects).

Against this backdrop, we compared the effects of introducing novel nouns and verbs on infants' performance in a series of experiments with monolingual 24-month-olds acquiring either English (Chicago), Mandarin (Beijing), or Korean (Seoul; Arunachalam, Leddon, Song, Lee, \& Waxman, in press; Arunachalam \& Waxman, 2011; Leddon et al., 2011). Infants from all three language groups viewed the same series of dynamic scenes (e.g., a girl petting a dog) while listening to a novel word, presented either as a novel noun (e.g., "The girl is petting the blick") or as a verb (e.g., "The girl is blicking the dog"). At test infants then viewed two new scenes, involving a change in either participant object (e.g., the girl petting a ball) or the action (e.g., the girl kissing the dog). Infants' performance with novel nouns was uniform and robust. They focused specifically on the test scene that depicted the same participant (e.g., dog), even if it was engaged in a different action (kissing). Infants' performance with novel verbs painted a different picture. Although they were sometimes able to map novel verbs to actions, their performance was considerably less robust and cross-linguistically more variable, echoing the variability of older children (Imai et al., 2005; Imai et al., 2008; Matsuo, Kita, Shinya, Wood, \& Naigles, 2012). Moreover, the linguistic contexts that best supported infants' verb learning differed systematically as a function of their native language and the way in which verbs in that language tend to be expressed. For example, in Korean (where noun phrases are typically dropped in situations like those in the test scenes), 24-month-olds were better able to map verbs to actions if the surrounding noun phrases (e.g., the girl; the dog) were dropped, but struggled if these were explicitly mentioned. In contrast, in English (where noun phrases typically are not dropped), infants were more successful mapping verbs to actions if the surrounding noun phrases were explicitly mentioned (Arunachalam \& Waxman, 2011; Arunachalam et al., in press; Leddon et al., 2011).

\section{SUMMARY}

This cross-linguistic study with infants marks a path that promises to bring us closer to addressing the long-standing debate concerning the impact of distinctly different languages on early language and conceptual development. We now know that by 24 months, infants acquiring a range of languages can successfully map novel nouns to object categories and novel verbs to event categories. This provides a foundation for tracing the developmental trajectory underlying noun and verb learning across languages. To discover whether and how the early acquisition of nouns and verbs is shaped by features of the ambient language, we must continue to focus research attention on young infants who are in the process of acquiring their native language.

This study also sheds light on the cross-linguistic status of the early noun advantage. In all the languages examined thus far, we see a robust ability to map novel nouns to object categories, but when it comes to mapping novel verbs to event categories, a different picture emerges. Infants have considerably more difficulty. Their ability to learn the meaning of a novel verb varies as a function of the particular language they are acquiring, and within a given language, it varies as a function of the particular linguistic contexts in which the verb appears (e.g., whether the surrounding noun phrases are mentioned explicitly or dropped).

This new cross-linguistic developmental evidence brings us closer to discovering the myriad forces that shape early noun and verb learning, and moves us beyond characterizing languages dichotomously — as either noun-friendly or verb-friendly — and urges us to adopt a more nuanced treatment of the syntactic, semantic, morphologic, and pragmatic properties of each language and the consequences of these properties on infants' acquisition of linguistic structure and meaning.

\section{REFERENCES}

Arunachalam, S., Leddon, E. M., Song, H., Lee, Y., \& Waxman, S. R. (in press). Doing more with less: Verb learning in Korean-acquiring 24-month-olds. Language Acquisition.

Arunachalam, S., \& Waxman, S. R. (2011). Grammatical form and semantic context in verb learning. Language Learning and Development, 7, 169-184.

Au, T. K., Dapretto, M., \& Song, Y. K. (1994). Input vs. constraints: Early word acquisition in Korean and English. Journal of Memory and Language, 33, 567-582. 
Bornstein, M. H., Cote, L. R., Maital, S., Painter, K., Park, S.-Y., Pascual, L., et al. (2004). Cross-linguistic analysis of vocabulary in young children: Spanish, Ditch, French, Hebrew, Italian, Korean, and American English. Child Development, 75, 111-1139.

Chan, C. C. Y., Tardif, T., Chen, J., Pulverman, R. B., Zhu, L., \& Meng, X. (2011). English- and Chinese-learning infants map novel labels to objects and actions differently. Developmental Psychology, 47, $1457-1471$.

Chemla, E., Mintz, T. H., Bernal, S., \& Christophe, A. (2009). Categorizing words using "frequent frames": What cross-linguistic analyses reveal about distributional acquisition strategies. Developmental Science, 12, 396-406.

Choi, S. (2000). Caregiver input in English and Korean: Use of nouns and verbs in book-reading and toy-play contexts. Journal of Child Language, 27, 69-96.

Choi, S., \& Gopnik, A. (1995). Early acquisition of verbs in Korean: A cross-linguistic study. Journal of Child Language, 22, 497-529.

Fisher, C., Hall, D. G., Rakowitz, S., \& Gleitman, L. (1994). When it is better to receive than to give: Syntactic and conceptual constraints on vocabulary growth. Lingua, 92, 333-375.

Gentner, D. (1982). Why nouns are learned before verbs: Linguistic relativity versus natural partitioning. In S. A. Kuczaj (Ed.), Language development: Vol. 2. Language, thought, and culture (pp. 301-334). Hillsdale, NJ: Erlbaum.

Gentner, D. (2006). Why verbs are hard to learn. In K. Hirsh-Pasek \& R. Golinkoff (Eds.), Action meets word: How children learn verbs (pp. 544-564). New York: Oxford University Press.

Gentner, D., \& Boroditsky, L. (2001). Individuation, relativity, and early word learning. In M. Bowerman \& S. Levinson (Eds), Language acquisition and conceptual development (pp. 215-256). New York: Cambridge University Press.

Gleitman, L. (1990). The structural sources of verb meanings. Language Acquisition: A Journal of Developmental Linguistics, 1, 3-55.

Gleitman, L. R., Cassidy, K., Nappa, R., Papafragou, A., \& Trueswell, J. C. (2005). Hard words. Language Learning and Development, 1, $23-64$.

Golinkoff, R. M., \& Hirsh-Pasek, K. (2008). How toddlers begin to learn verbs. Trends in Cognitive Sciences, 12, 397-403.

Gopnik, A., Choi, S., \& Baumberger, T. (1996). Cross-linguistic differences in early semantic and cognitive development. Cognitive Development, 11, 197-227.

Imai, M., Haryu, E., \& Okada, H. (2005). Mapping novel nouns and verbs onto dynamic action events: Are verb meanings easier to learn than noun meanings for Japanese children? Child Development, 76, 340-355.

Imai, M., Li, L., Haryu, E., Okada, H., Hirsh-Pasek, K., Golinkoff, R., et al. (2008). Novel noun and verb learning in Chinese-, English-, and Japanese-speaking children. Child Development, 79, 979-1000.

Katerelos, M., Poulin-Dubois, D., \& Oshima-Takane, Y. (2011). A cross-linguistic study of word-mapping in 18- to 20-month-old infants. Infancy, 16, 508-534.

Kauschke, C., Lee, H., \& Pae, S. (2007). Similarities and variation in noun and verb acquisition: A crosslinguistic study of children learning German, Korean, and Turkish. Language and Cognitive Processes, 22, 1045-1072.

Kersten, A. W., \& Smith, L. B. (2002). Attention to novel objects during verb learning. Child Development, 73, 93-109.

Kim, M., McGregor, K. K., \& Thompson, C. K. (2000). Early lexical development in English-and Korean-speaking children: Language- general and language-specific patterns. Journal of Child Language, $27,225-254$.

Leddon, E. M., Arunachalam, S., Waxman, S. R., Fu, X., Gong, H., \& Wang, L. (2011). Noun and verb learning in Mandarin-acquiring 24-month-olds. Online Proceedings Supplement of the 35th Annual Boston University Conference on Language Development.

Lee, J. N., \& Naigles, L. R. (2005). The input to verb learning in Mandarin Chinese: A role for syntactic bootstrapping. Developmental Psychology, 41, 529-540.

Ma, W., Golinkoff, R. M., Hirsh-Pasek, K., McDonough, C., \& Tardif, T. (2009). Imageability predicts the age of acquisition of verbs in Chinese children. Journal of Child Language, 36, 405-423.

Maratsos, M., \& Chalkley, M. (1980). The internal language of children's syntax: The ontogenesis and representation of syntactic categories. In K. Nelson (Ed.), Children's language (Vol. 2, pp. 127-214). New York: Gardner Press.

Matsuo, A., Kita, S., Shinya, Y., Wood, G. C., \& Naigles, L. (2012). Japanese two-year-olds use morphosyntax to learn novel verb meanings. Journal of Child Language, 39, 637-663.

McDonough, C., Song, L., Hirsh-Pasek, K., Golinkoff, R. M., \& Lannon, R. (2011). An image is worth a thousand words: Why nouns tend to dominate verbs in early word learning. Developmental Science, 14, 181-189.

Mintz, T. H. (2003). Frequent frames as a cue for grammatical categories in child directed speech. Cognition, 90, 91-117.

Nisbett, R. E., Peng, K., Choi, I., \& Norenzayan, A. (2001). Culture and systems of thought: Holistic versus analytic cognition. Psychological Review, 108, 291-310.

Oshima-Takane, Y., Ariyama, J., Kobayashi, T., Katerelos, M., \& Poulin-Dubois, D. (2011). Early verb learning in 20-month-old Japanese-speaking children. Journal of Child Language, 38, 455-484.

Sandhofer, C. M., Smith, L. B., \& Luo, J. (2000). Counting nouns and verbs in the input: Differential frequencies, different kinds of learning? Journal of Child Language, 27, 561-585.

Stoll, S., Bickel, B., Lieven, E., Paudyal, N. P., Banjade, G., Bhatta, T. N., et al. (2012). Nouns and verbs in Chintang: Children's usage and surrounding adult speech. Journal of Child Language, 34, $284-321$.

Tardif, T. (1996). Nouns are not always learned before verbs: Evidence from Mandarin speakers' early vocabularies. Developmental Psychology, 32, 492-504.

Tardif, T., Fletcher, P., Liang, W., Zhang, Z., Kaciroti, N., \& Marchman, V. A. (2008). Baby's first 10 words. Developmental Psychology, 44, 929-938.

Tardif, T., Gelman, S. A., \& Xu, F. (1999). Putting the "noun bias" in context: A comparison of English and Mandarin. Child Development, 70, 620-635.

Tardif, T., Shatz, M., \& Naigles, L. (1997). Caregiver speech and children's use of nouns versus verbs: A comparison of English, Italian, and Mandarin. Journal of Child Language, 24, 535-565.

Waxman, S. R., \& Lidz, J. (2006). Early word learning. In D. Kuhn \& R. Siegler (Eds), Handbook of child psychology (Vol. 2, 6th ed., pp. 299-335). Hoboken, NJ: Wiley.

Waxman, S. R., Lidz, J. L., Braun, I. E., \& Lavin, T. (2009). Twenty four-month-old infants' interpretations of novel verbs and nouns in dynamic scenes. Cognitive Psychology, 59, 67-95.

Yuan, S., \& Fisher, C. (2009). "Really? She blicked the baby?" Twoyear-olds learn combinatorial facts about verbs by listening. Psychological Science, 20, 619-626. 